\title{
Computer wireless network integration technology in equipment state data collection
}

\author{
Lixin $M a^{*} \mathbb{D}$
}

\section{${ }^{*}$ Correspondence:}

sdmalixin@163.com

School of New Generation

Information Technology

Industry, Shandong

Polytechnic, Jinan, China

\begin{abstract}
The paper introduces a handheld integrated power data acquisition and analysis equipment based on a computer wireless network mobile platform, an intelligent transportation inspection box. The intelligent transportation inspection box acts as a bridge between the transportation inspection work site side and the power grid company center to realize data and resource sharing between the site and center sides. The application of intelligent operation and inspection boxes based on computer wireless network integration technology is essential for improving the professional and intelligent level of on-site operation and maintenance, improving the efficiency of operation and inspection work and the ability to control equipment status, strengthening state maintenance and auxiliary decision-making, and promoting the construction of smart grids significance.
\end{abstract}

Keywords: Wireless network integration technology, Intelligent transportation inspection box, Power system, Equipment status, Data collection

\section{Introduction}

Under the irreversible application trend of new technologies such as interconnection, cloud computing, big data, and the Internet of Things, the original technology and management mode of power grid companies have been significantly impacted. On-site operation and maintenance intelligence and management and control are essential areas for applying new information and communication technologies and the construction of management and control platforms [1]. It is urgent to research the intelligent system of on-site operations to achieve the standardized collection of on-site data, standardized guidance of on-site operations, and multi-dimensional real-time equipment status analysis. Data upload, on-site and center-side data, and analysis algorithms are mutually shared, improving on-site operations' efficiency and providing system support for developing new information technologies on-site and on-site management and control.

However, in the power operation and maintenance service, equipment status data such as live detection data are mostly manually entered, which has problems such as high operation and maintenance costs and low work efficiency. Therefore, we need to establish an integrated system of intelligent field operation and operation and maintenance of substation equipment under the new situation through new information and 
communication technologies such as the Internet of Things, mobile internet, and cloud computing [2]. We have developed a handheld integrated power data acquisition and analysis device, an intelligent transportation and inspection box. We build a field operation platform to realize field operation standardization and data standardization and realize the mutual sharing of data and analysis algorithms on the field and center sides [3].

The platform's design can improve on-site data analysis capabilities, provide support for on-site operations and advanced data applications, and strengthen remote control and real-time technical support capabilities for on-site operation and maintenance.

\section{Methods}

Although the State Grid's operation, inspection and maintenance process is highly informative, it is supported by Production Management System (PMS) and Operations Management System (OMS) systems. However, on the field side, equipment status information is scattered in different systems. Not only is there a lack of interconnection between them and the PMS and OMS on the central side, but there are barriers between the systems, which quickly form "information islands."

To alleviate the current situation of information asymmetry between the center side and the field side and solve the problems of poor on-site operation and maintenance data, non-standard workflow, weak data analysis ability, and disconnection from the center side [4], the paper uses the Internet of Things, mobile Internet, and New information and communication technologies such as cloud computing have developed intelligent transportation and inspection boxes as a bridge for real-time data exchange between the center side and the field side.

\subsection{Collection strategy}

Generally, when the equipment status changes rapidly because of equipment failures, etc., it often causes the accelerated equipment status changes. To this end, we need to give an allowable range of equipment state value. Assume that the minimum allowable change of equipment state parameters is $\delta_{\min }$ and the maximum allowable change is $\delta_{\max }$. The primary acquisition strategy of the new adaptive method is as follows:

(1) When the change of the monitoring value before and after the equipment status is less than $\delta_{\min }$, it means that the collection accuracy has far exceeded the user's needs. At this time, the collection frequency can be reduced, and the collection and storage of low-value data can be reduced. (2) When the equipment changes, the monitoring value before and after the state is more significant than $\delta_{\max }$, it means that the acquisition accuracy can no longer meet the user needs [5]. At this time, the acquisition frequency can be increased to improve the system's ability to capture changes in the device state. (3) When the device state changes before and after the monitoring value. When the amount is between $\delta_{\min }$ and $\delta_{\max }$, it indicates that the acquisition accuracy meets the user's needs. The original acquisition frequency can be maintained at this time.

The above strategy $\delta_{\min }$ and $\delta_{\max }$ need to be determined according to the user's accuracy requirements for the monitoring value and combined with historical data statistics. For example, the minimum allowable collection interval $\Delta T_{\min }$ can be used to collect a certain amount of raw data at equal intervals, and the status monitoring can be obtained 
by statistics the average change of the value and then take the integer multiple of the average change as the reference value of $\delta_{\min }$ and $\delta_{\max }$. Of course, this should also be combined with the user's accuracy requirements.

\subsection{Algorithm design}

Assume that $t_{n}, f_{n}$ are the time of the nth acquisition and the obtained device state parameter value; $\Delta T$ is the system acquisition interval, which satisfies $\Delta T_{\min } \prec \Delta T \prec \Delta T_{\max }$. Among them, the value of $\Delta T_{\min }$ depends on the maximum acquisition speed of the system itself and is also affected by the network load and the network load [6]. Throughput capacity and user requirements; the value of $\Delta T_{\max }$ depends on the user's minimum requirements for the system's collection frequency or monitoring value accuracy. To achieve the above collection strategy, how to define the change of the monitoring value before and after the device state is the key, the traditional change the definition method is

$$
\Delta f=\left|f_{n}-f_{n-1}\right|
$$

The definition method of Formula (1) will inevitably lead to a result. As long as the device changes, the system will change the collection interval, even if the change is instantaneous and short-lived as instantaneous impact. This article uses a moving average method to describe the change in the device's state to overcome the collection interval for possible disorderly changes. At this time, for the change in the monitored value, two situations need to be considered [7]:

(1) The monitored value deviated from the center value instantaneously but immediately returned to the vicinity of the center value. If there is an accidental instantaneous impact, the collection interval at this time does not need to be changed. To eliminate this interference factor, the amount of state change of the device can be defined for

$$
\Delta f=\left(\left|f_{n}-f_{n-1}\right|+\left|f_{n+1}-f_{n-1}\right|+\ldots\left|f_{n+k-1}-f_{n-1}\right|\right) / k
$$

In the formula, $\mathrm{k}$ is the number of monitoring points considered in the moving average, which needs to be determined according to the characteristics of the user's equipment and needs. If the interference is minor, a small value can be used; otherwise, an enormous value should be considered. Equation (2) The comparison benchmark is the $\mathrm{n}-1$ th data collection point. The value of a certain point in the subsequent $\mathrm{k}$ collection points is not enough to change the system's collection interval.

(2) The monitoring value has an instantaneous mutation based on the original center value, and then it runs stably around the new center value immediately. As a sudden increase in load, it is very likely to cause this situation, and the collection interval also does not need to be changed. To this end, the amount of state change of the device can be defined as

$$
\Delta f=\left(\left|f_{n}-f_{n-1}\right|+\left|f_{n+1}-f_{n-1}\right|+\ldots\left|f_{n+k-1}-f_{n+k-2}\right|\right) / k
$$

The comparison basis of Formula (3) is the previous point of the two adjacent data collection points instead of keeping the $\mathrm{n}$-1th point unchanged. Under this constraint, only the difference between the adjacent points of the equipment state monitoring value 
in continuous over-tolerance, the system's collection interval may be changed. In actual operation, the above two situations must be considered at the same time.

In summary, the steps of the adaptive acquisition algorithm in this article are as follows: (1) Select the initial values of parameters $\delta_{\min }, \delta_{\max }, \mathrm{k}$ and $\Delta T$ (2) Collect data at intervals of $\Delta T$. (3) During the acquisition process, every step must be Judge whether Formula (1) is in $\left[\delta_{\min }, \delta_{\max }\right]$, if it is, keep the original frequency and continue to collect; if it is out of tolerance, then use Formulas (2) and (3) to perform the out-of-tolerance judgment at the same time. (4) Use Formula (2), (3) When performing out-of-tolerance judgment, as long as one of the calculation results falls within $\left[\delta_{\min }, \delta_{\max }\right]$, the system will keep the original frequency and continue to collect; if all are out of the allowable range, perform step (5). (5) If you use the formula, the calculation results of (1)-(3) are all less than $\delta_{\min }$, then reduce the acquisition frequency; if the three are more significant than $\delta_{\max }$, then increase the acquisition frequency; otherwise, keep the original acquisition frequency.

In the above steps, the initial collection interval $\Delta T$ needs to be selected according to the device's characteristics. Generally speaking, as the device's use time increases, the instability of its operating state will increase accordingly, so the value of $\Delta T$ is increased appropriately. During the entire collection process, $\Delta T$ will increase or decrease as the device status changes. Taking into account the convenience of operation, within the interval constraint, an integer multiple of the minimum collection interval $\Delta T_{\min }$ can be used to define the value range of $\Delta T$. That is to meet:

$$
\left\{\begin{array}{l}
\Delta T \in\left\{\Delta T_{\min }, \Delta T_{\min }, \ldots, \Delta T_{\min }\right\} \\
\Delta T \leq \Delta T_{\max }
\end{array}\right\}
$$

\section{The design of the intelligent transportation and inspection box}

\subsection{Intelligent transportation inspection box and integrated operation and maintenance management and control platform}

Figure 1 is a power system operation and maintenance integrated management and control platform equipped with an intelligent operation and inspection box proposed in this paper. This system combines information system technology, mobile platform technology, Internet of Things technology, and big data technology to revolutionize the operation and inspection of equipment. The intelligent transportation inspection box and the power detection equipment are connected through a wireless network and communicate through a unified communication protocol on the field side. The transportation inspection personnel performs any transportation inspection, such as live detection, intelligent inspection or preventive testing, etc. The big data and computing resources on the grid company's central site can also interact with the on-site operation and maintenance activities through the operation inspection box. For example, the advanced application on the center side can analyze the data uploaded on the field side and perform matching and analysis through the case library. If abnormal data or emergency equipment failure is found, the field side can be directly notified to intervene in the field side's operation and maintenance activities. When the on-site operation and maintenance personnel encounter technical difficulties, they can also directly call the power grid company through the 

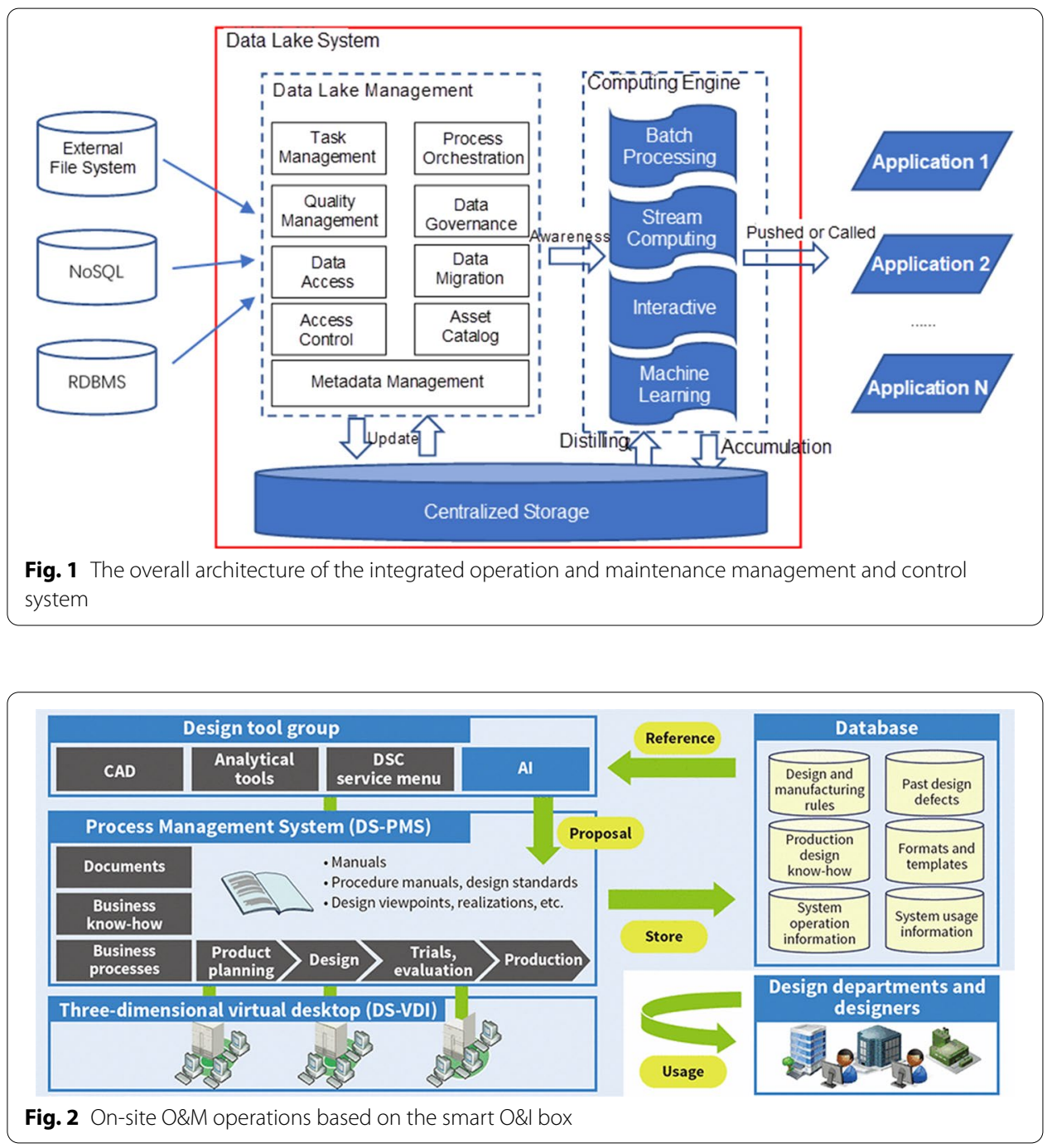

video conference module of the inspection box to organize temporary video conferences and obtain on-site guidance from experts on the power grid company's central site.

\subsection{Operation and maintenance workflow using smart transportation and inspection boxes}

During the inspection process, it is necessary to ensure the real-time connection between the personnel and equipment at the inspection site and the integrated management and control platform, big data and experts on the center side. On the one hand, the data of the field test can be uploaded in time. On the other hand, the center side's decision can be sent and pushed in real-time to realize resource sharing, complementary advantages, and complete daily operation and inspection work in the interaction [8]. The schematic diagram of operation and maintenance is shown in Fig. 2.

On the central site of the power grid company, managers use the integrated management and control platform on the central side to formulate operation and maintenance 
tasks, such as which substations need to be patrolled and which operation and maintenance tests need to be carried out.

The intelligent operation and inspection box establish an independent WIFI wireless network, thereby establishing an automatic wireless network connection with various power detection equipment [9]. According to the operation guidance prompted by the intelligent operation and inspection APP, the operation and inspection personnel operate the corresponding power detection equipment and carry out the operation and maintenance test step by step. The experimental data are automatically stored in the operation and inspection box. After the test is completed, the operation and maintenance personnel only need to touch the tablet interface to generate a test report and realize one-click data upload automatically [10]. The use of intelligent transportation inspection boxes can significantly reduce the labor intensity of management personnel and operation and maintenance personnel. Operation and maintenance personnel only need to follow the APP prompts to perform corresponding operations, and the consistency and reliability of the uploaded data can also be effectively obtained.

\section{The software and hardware architecture of the intelligent transportation inspection box}

\subsection{Hardware architecture}

Considering performance and energy consumption comprehensively, the intelligent transportation inspection box adopts the OMAPTM5432 platform, and the CPU adopts the Exynos5250 of the ARMCorTex-A15 framework, primary frequency is up to $1.7 \mathrm{GHz}$. Exynos5250 adopts the 32-nm HKMG process and is equipped with Mali-604GPU, which has powerful performance and meets high-end integer and floating-point computing performance. Figure 3 is the hardware circuit of the intelligent transport inspection box.

\subsection{Software architecture}

The device's software design includes two parts: a data acquisition program and a data analysis software program. The data acquisition program is based on the DSP data acquisition module. We use the TMS320C2XX standard assembly language to compile. The code execution efficiency is high, and it can meet real-time requirements. It mainly operates on the hardware to complete the data acquisition and upload function. The data analysis software is based on an embedded Linux real-time multitasking operating system. An embedded real-time operating system is a multitasking operating system and a development platform for embedded application software. Linux is an open Unix system version, which belongs to "free software," and programs and their source codes can be obtained freely and used freely. The software adopts the cross-platform $\mathrm{C}++$ Graphical User Interface (GUI) toolkit QT development analysis tool, which has the characteristics of multitasking, multi-user, device independence and sound portability and has a good user interface. The embedded Linux operating system on the device is cut according to the specific hardware resources, and only the necessary functional modules are retained. The kernel is refined, the resources are small, and the functions are perfect. Objectoriented, modular design and other ideas are used in the program's programming to 


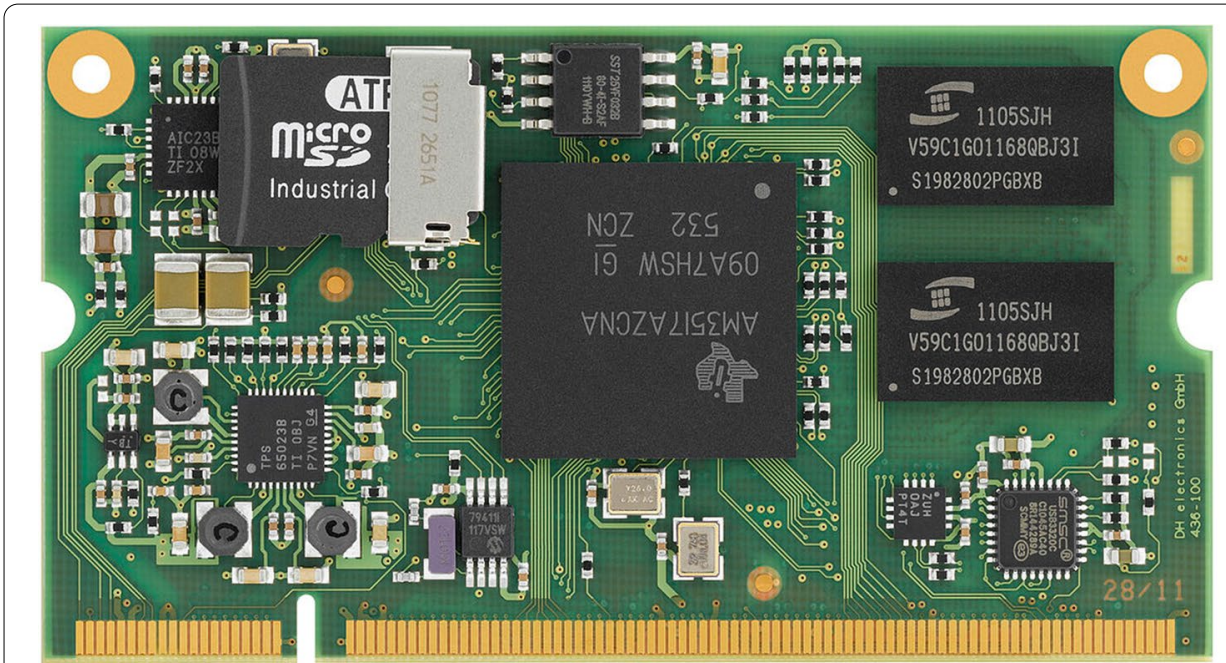

Fig. 3 The hardware circuit of the smart shipping inspection box

support multithreading and multitasking. Figure 4 is an architecture diagram of the software system of the transport inspection box.

\subsubsection{Data acquisition module program}

The data collection program's primary function is the setting and judging of the starting conditions, data collection, and data upload. The sampling frequency and starting conditions of the device can be defined, and the recording time can be set. There are three start recording conditions: continuous timing recording, circular recording, and conditional recording. Timing continuous recording refers to the setting device to start sampling at a particular time and perform the continuous recording of the data for a certain period; circular recording first sets the length of the circular recording data as required and then performs continuous circular recording of the data, data recording. When the

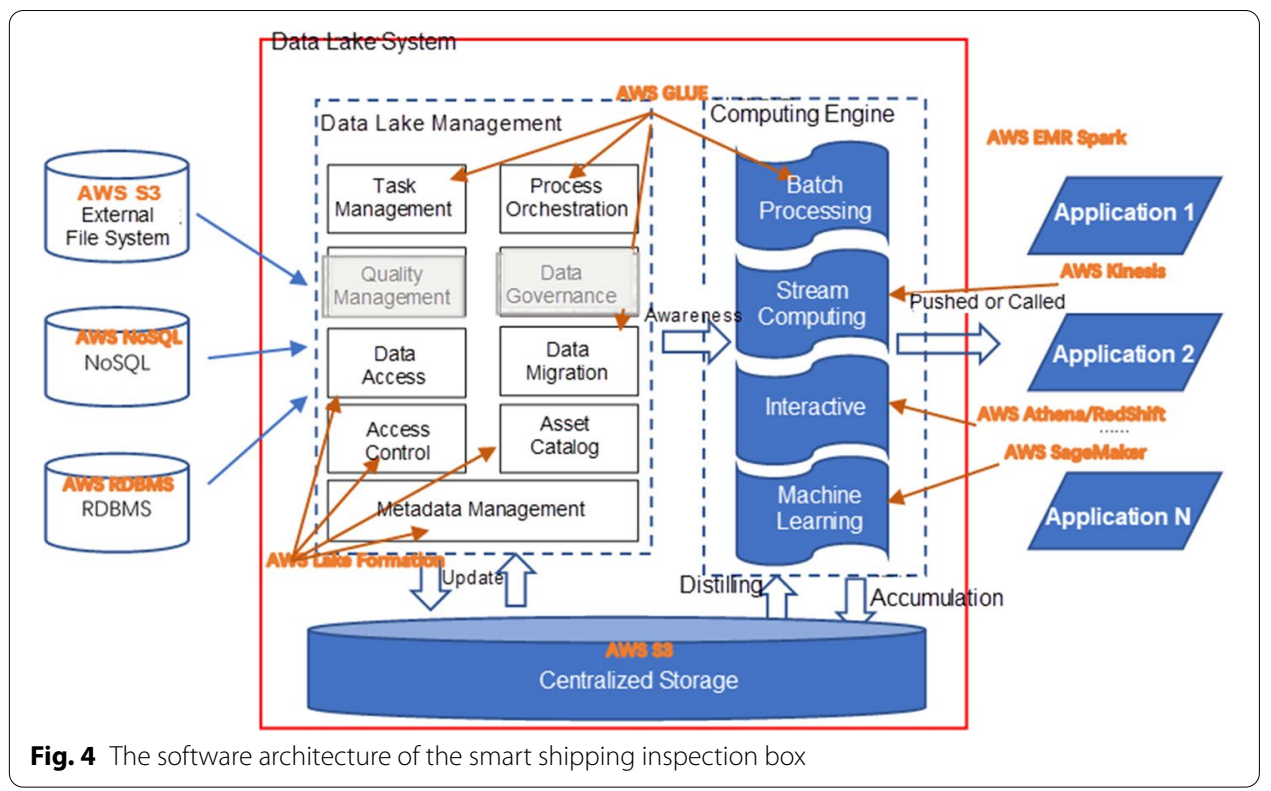


set length is reached, the latest data will automatically overwrite the old data, to ensure that the recorded data are the latest; the conditional recording is to set the device to start the wave recording under certain conditions automatically. Start by conditions can be divided into the following ways:

(a) Sudden change start, including voltage change and current change start; (b) Positive sequence start, including overvoltage, low voltage, and overcurrent start during regular operation; (c) The negative sequence starts using three-phase voltage, three-phase voltage, three-phase. The phase current's sampling value is calculated to obtain the negative sequence voltage and the negative sequence current, including the negative sequence overvoltage and the negative sequence overcurrent start; (d) The zero-sequence start, the zero-sequence voltage, and the zero-sequence current are obtained by direct sampling. Starting conditions include zero-sequence voltage and zero-sequence sudden current changes and steady-state over-start; (e) DC start, other forms of physical quantities are transformed into DC voltage by the sensor, starting conditions include over and under start; (f) Frequency Start, including high frequency, low frequency and frequency change rate start. The data acquisition process is divided into three stages: about ten cycles of data are collected before the fault, about $5 \mathrm{~s}$ of data is collected after the fault starts, and finally $3-5 \mathrm{~s}$ of data under normal conditions. The sampling frequency is adjustable, and the three stages use different sampling frequencies.

\subsubsection{Data analysis program}

The data analysis software mainly completes the device's function setting and the comprehensive analysis of the data. The program adopts an object-oriented modular design, and each functional module is independent of the other. The system functions include the following points: (a) Parameter settings. Mainly set the recording mode, analog parameter, and switch parameter of the device. (b) File management. Provide query and copy functions of recorded data files. (c) Regarding the device. Introduce the device's performance characteristics and some simple operation methods equivalent to the simple instructions for the device. (d) Date and time. The current date and time are displayed and refreshed every second.

The data analysis function includes the following points: (a) Waveform display. Display the waveform of the collected analog quantity, analyze and edit the waveform, and zoom in and out of the amplitude and time axis. The waveform is displayed by channel, and up to 6 channel signal waveforms can be displayed at a time. (b) Harmonic analysis. Provides full-cycle Fourier and fast Fourier algorithms extract the fundamental wave and each harmonic component and obtain a list of the fundamental part, imaginary part, compelling value, phase angle and proportion, and displays each in the form of a histogram. The percentage of sub-harmonics. (c) DC transient quantity. Extract the attenuated DC component of the signal, display the attenuated DC component's waveform, and calculate the initial value and the attenuation time constant. (d) Symmetrical component. The change of positive, negative, zero-sequence voltage and current before and after the fault is displayed in a vector diagram. (e) Calculation function. It can perform calculations such as addition, subtraction, multiplication, and division, divided into instantaneous value and vector value calculation. The instantaneous value calculation can calculate the instantaneous 
quantity and output the waveform; the vector value calculation can calculate the fundamental wave and each harmonic according to the natural part, imaginary part, amplitude, and phase angle. (f) Power quality. Provides basic parameters of power quality such as voltage, current, active power, reactive power, frequency, etc., and can analyze power quality indicators such as harmonics, three-phase voltage unbalance, and voltage flicker power system. (g) Power spectrum analysis. The Fourier transform of random signals does not exist. The frequency-domain analysis of random signals is no longer a simple spectrum but a power spectrum. The software provides the power spectrum analysis function, which can analyze the signal power conversion with frequency in the unit frequency band of the analog signal. (h) Random signal. Providing special tools for random signal analysis, separate the transient quantities in the signal, and extract the characteristic components as the basis for fault calculation and signal analysis. (i) Extended functions. Providing a standard program interface for the expansion of software functions.

\subsection{Centre-side integrated operation and maintenance platform}

The center-side integrated operation and maintenance platform makes full use of the power grid company's center side's data advantages to conducting unified management and guidance of on-site operation and maintenance activities. It collects the operation and maintenance test data uploaded from the scene by the operation inspection box through the network and stores it in the database. Simultaneously, according to the onsite operation situation, decision-making support is made and sent to the on-site side through the network to guide the operation and maintenance personnel [11]. The central-side system is set with a standardized advanced application framework.

\subsection{Communication protocol with power test equipment}

There are many types of power test equipment. The communication protocol between the intelligent transportation inspection box and them must have a certain degree of normalization, openness, and forward-looking. The design of the communication protocol is based on the Message Queuing Telemetry Transmission (MQTT) protocol. In terms of specific data encoding, the communication protocol contains the test equipment's information and contains the information of the test experiment, which are, respectively, calibrated with tags. The existing mainstream power test equipment generally has a communication interface, but it does not necessarily comply with the intelligent transport inspection box's communication protocol. To solve this problem, the test equipment must be modified. The specific plan is to install an intelligent wireless communication module based on the original equipment's communication interface. The intelligent wireless communication module is used as middleware to translate the original communication mode into a communication protocol that meets the requirements. Of course, the intelligent transformation of existing equipment is not accomplished overnight and needs to be completed gradually. The transformation of multiple pieces of equipment such as infrared imaging and UHF partial discharge detectors produced by NARI has been completed. 


\section{Results and discussion}

The use of intelligent operation and inspection boxes can significantly improve operation and maintenance work efficiency, the reliability, and standardization of data. This article takes the daily work of substation live operation and maintenance as an example. Briefly, it introduces the working examples of the intelligent operation inspection box and the integrated operation and maintenance management system.

In the integrated operation and maintenance system, users mainly have two roles according to labor division. One is the power grid company's project manager, who comprehensively manages the substation inspection project, assigns and issues tasks for on-site operation and maintenance personnel, checks the data collected on-site, and supervises operation completion and maintenance tasks. The second is the on-site operation and maintenance team. Under the project manager's supervision, they use APP to perform task download and data upload operations on the live detection test, realize data interaction between the scene and the client and display and feedback the client's live detection information in real-time. Their operational inspection workflow is shown in Fig. 5.

\subsection{Working example of project manager}

The project manager's working place is on the central site of the power grid company. $\mathrm{He}$ can log in to the integrated operation and maintenance management software website through the Web, establish operation inspection tasks, and view the operation inspection results. The left half of Fig. 5 is the workflow for the project manager to establish an operational inspection task. First, he enters the account password and logs in to the integrated operation and maintenance platform, then enter the operation and inspection item information under the web interface, establish a specific operation and inspection item, give the item's importance, and assign the operation and inspection personnel to the item.

When the inspection project is released, the system will push a message to the inspection team. After receiving the task, the transportation inspection team will take the test equipment and transportation inspection box to the transportation inspection site [12]. At this time, the project manager monitors each operation and maintenance team's work at any time through the integrated operation and maintenance system.

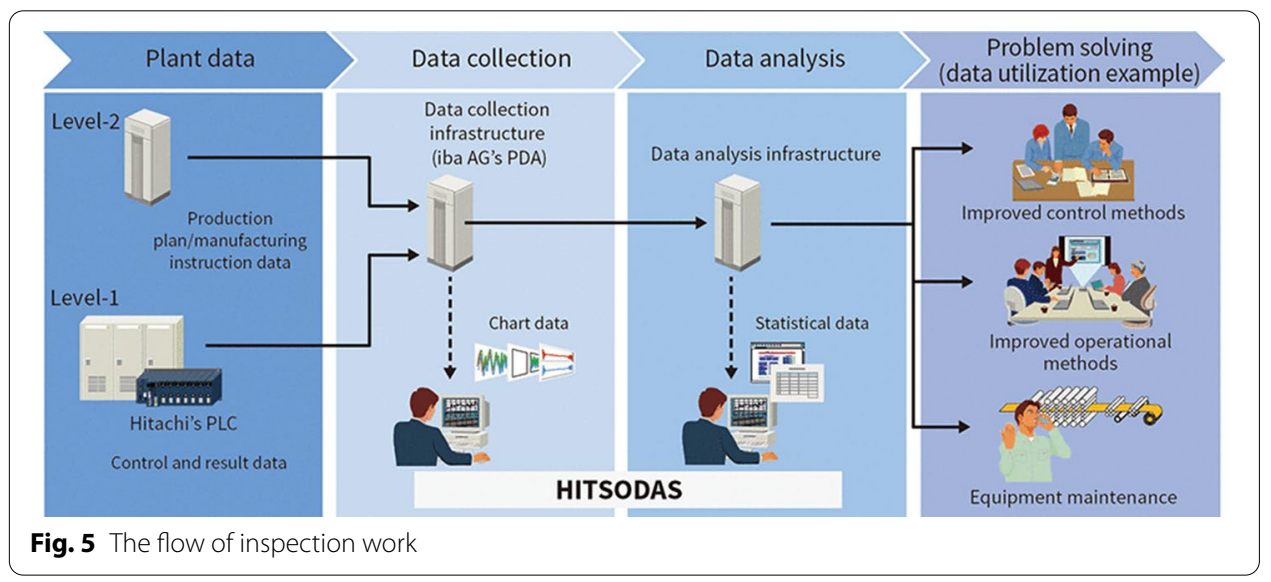




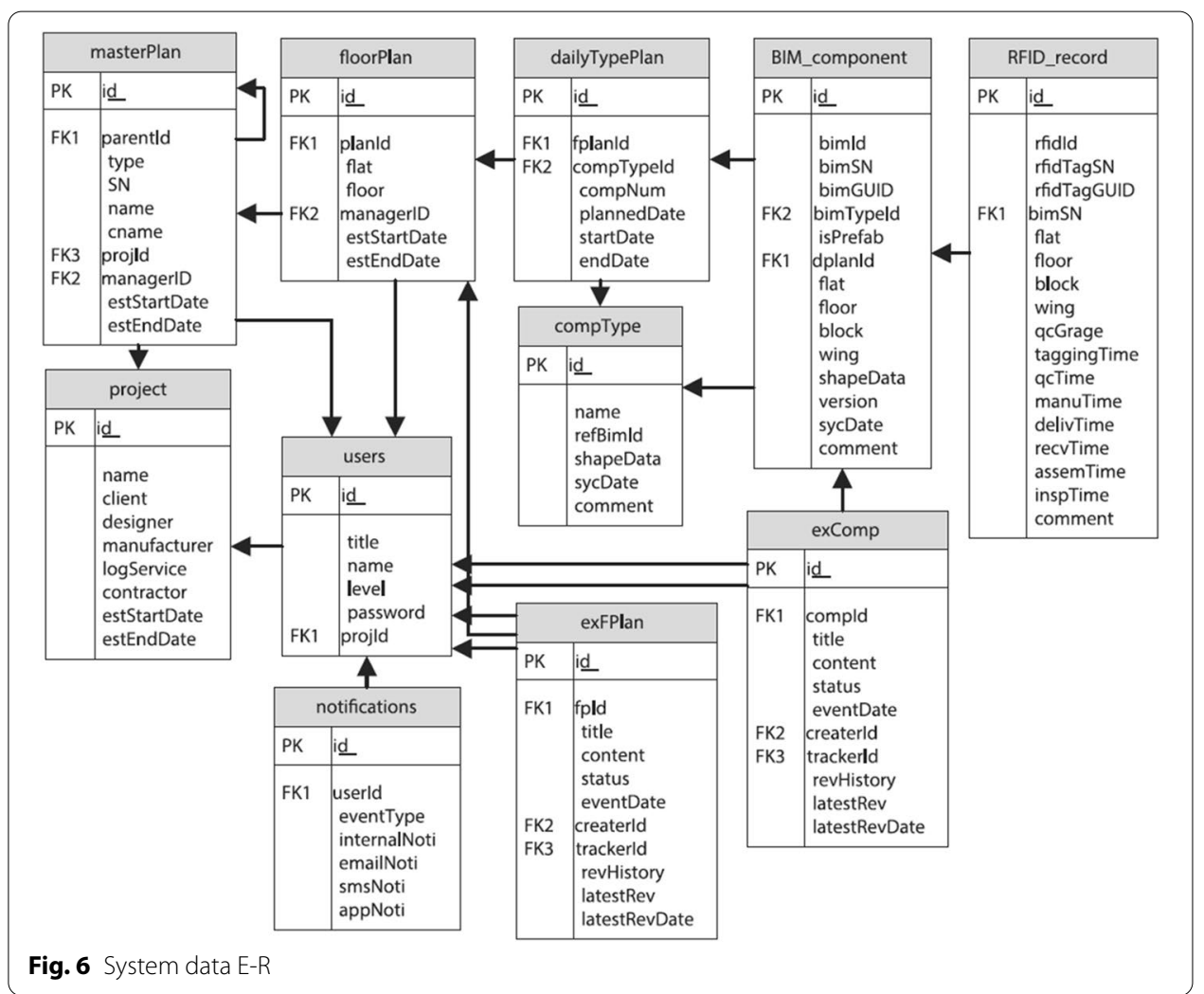

5.2 Working example of the transportation inspection team

After receiving the operation and maintenance task, the transportation inspection team brings the testing equipment, smart transportation inspection box, and tablet computer to the corresponding workplace. After the operation and maintenance team has placed the excellent transportation inspection equipment and the intelligent transportation inspection box, they can turn on the tablet and run the transportation inspection APP. The transportation inspection APP and the intelligent transportation inspection box are networked through the WIFI network for data communication. The transportation inspection APP calls the intelligent transportation inspection box to download transportation inspection tasks from the center side through the mobile network and display them on the tablet.

According to the task requirements, the transportation inspection team uses the test equipment to carry out the inspection task. The APP will also guide the operation and inspection personnel according to the progress of the task.

Taking infrared detection as an example, the operation and maintenance team can use the APP to operate the infrared imager to take infrared photographs of the electrical equipment under inspection. The infrared imager is automatically connected to the transportation inspection box, and the photographs taken are automatically stored in the intelligent transportation inspection box's file system. When the infrared inspection work is over, the transportation inspector can use the APP to browse the test data, select the appropriate infrared image, and enter it into the system as the test data. Figure 6 shows the system data E-R. 
According to the APP prompts, the inspection personnel can inspect and test items step by step. Suppose they encounter any problems in the process. In that case, they can call the integrated management and control platform on the power grid center side through the intelligent inspection box at any time. The management personnel on the center side receive the call request, select the corresponding expert according to the problem's characteristics, and use the remote video conference method to provide realtime guidance for the operation and maintenance personnel to help them solve the problem [13]. In this way, experts do not need to go to the site in person, saving their time, and limited experts can serve more operation and maintenance teams.

When all the operation and inspection test tasks are completed, the intelligent operation and inspection box will automatically generate a test report and display it on the man-machine interface of the APP. The operation and maintenance team checks the test report's data, and if it is confirmed that it is correct, tap the "submit task" button to upload the test report to the database on the center side with one click.

\subsection{Advantages of using smart transport inspection box}

Judging from the working example, using the intelligent operation inspection box for the power system's operation and maintenance has the following advantages. (1) Simplify the work of operation and maintenance project managers. The project manager only needs to manage and issue operation and maintenance projects and tasks through the Web platform. When necessary, organize experts to provide technical guidance for on-site operation and maintenance through telephone conferences. (2) Reduce the work intensity of operation and maintenance personnel and improve labor efficiency. In on-site operation and maintenance, all equipment is connected to the intelligent operation and inspection box. Unified management, unified data upload, operation, and maintenance personnel do not need to record test data manually. (3) Reduce the requirements for the quality of operation and maintenance personnel. The intelligent transportation and inspection APP guides the operation of the transportation and inspection personnel throughout the process. When encountering technical difficulties, the video conference function of the intelligent transportation and inspection box can be used at any time to call the experts on the power grid center side for real-time guidance. (4) Data storage and management are more standardized. All data are collected uniformly through intelligent transportation and inspection boxes and are stored and uploaded centrally. The consistency, reliability, and uniformity of the data are well guaranteed.

\section{Conclusion}

This paper presents the design and implementation of an intelligent power system inspection box based on computer wireless network integration technology. The equipment design scheme makes full use of the embedded system's simple hardware structure, high efficiency, and strong pertinence. The software has the characteristics of real-time, multitasking, and small hardware resources. It combines the traditional data acquisition system and data analysis system into one. The data analysis software adopts an objectoriented design method, the software design is reasonable, the function is perfect, and it is convenient to use. It can adapt to the new needs of modern power systems development and have achieved pleasing operating results. 


\section{Abbreviations}

APP: Application; PMS: Production Management System; OMS: Operations Management System; WIFI: Wireless Fidelity: Gcc: GNUCCompiler; RDBMS: Relational Database Management System; USB: Universal Serial Bus.

\section{Acknowledgements}

The author would like to express his sincere thanks to the referees for the careful reading and their important comments which helped improve the original paper.

\section{Authors' contributions}

The authors read and approved the final manuscript.

\section{Funding}

None.

Availability of data and materials

Not applicable.

\section{Declarations}

\section{Competing interests}

The authors declare that they have no competing interests.

Received: 12 April 2021 Accepted: 1 July 2021

Published online: 13 July 2021

\section{References}

1. A. Al Rashdan, S. St. Germain, Methods of data collection in nuclear power plants. Nucl. Technol. 205(8), 1062-1074 (2019)

2. G. Han, X. Long, C. Zhu, M. Guizani, W. Zhang, A high-availability data collection scheme based on multi-AUVs for underwater sensor networks. IEEE Trans. Mob. Comput. 19(5), 1010-1022 (2019)

3. A. Shamayleh, M. Awad, J. Farhat, loT based predictive maintenance management of medical equipment. J. Med. Syst. 44(4), 1-12 (2020)

4. D. Zhou, Z. Yan, G. Liu, M. Atiquzzaman, An adaptive network data collection system in sdn. IEEE Trans. Cogn. Commun. Netw. 6(2), 562-574 (2019)

5. P. Wang, X. Liu, Z. Han, Multi-parameter online optimization algorithm of BP neural network algorithm in Internet of Things service. Neural Comput. Appl. 33(2), 505-515 (2021)

6. G. Han, Z. Tang, Y. He, J. Jiang, J.A. Ansere, District partition-based data collection algorithm with event dynamic competition in underwater acoustic sensor networks. IEEE Trans. Ind. Inf. 15(10), 5755-5764 (2019)

7. X. Feng, F. Yan, X. Liu, Study of wireless communication technologies on Internet of Things for precision agriculture. Wirel. Pers. Commun. 108(3), 1785-1802 (2019)

8. D. Liu, Y. Xu, J. Wang, J. Chen, K. Yao, Q. Wu, A. Anpalagan, Opportunistic UAV utilization in wireless networks: Motivations, applications, and challenges. IEEE Commun. Mag. 58(5), 62-68 (2020)

9. A. El Attaoui, M. Hazmi, A. Jilbab, A. Bourouhou, Wearable wireless sensors network for ECG telemonitoring using neural network for features extraction. Wirel. Pers. Commun. 111(3), 1955-1976 (2020)

10. J. Mei, X. Wang, K. Zheng, Intelligent network slicing for V2X services toward 5G. IEEE Netw. 33(6), 196-204 (2019)

11. Z. Lv, B. Hu, H. Lv, Infrastructure monitoring and operation for smart cities based on loT system. IEEE Trans. Ind. Inf. 16(3), 1957-1962 (2019)

12. S.F. Mjolsnes, R.F. Olimid, Private identification of subscribers in mobile networks: status and challenges. IEEE Commun. Mag. 57(9), 138-144 (2019)

13. Q. Qin, Development and realization of a computer monitoring system based on a power network. Recent Adv. Electr. Electron. Eng. (Former. Recent Patents Electr. Electron. Eng.) 14(1), 59-66 (2021)

\section{Publisher's Note}

Springer Nature remains neutral with regard to jurisdictional claims in published maps and institutional affiliations.

\section{Submit your manuscript to a SpringerOpen ${ }^{\circ}$ journal and benefit from:}

- Convenient online submission

- Rigorous peer review

- Open access: articles freely available online

- High visibility within the field

- Retaining the copyright to your article

Submit your next manuscript at $>$ springeropen.com 